\title{
E1 verbum cordis formabile agustiniano y la imagen trinitaria en el hombre
}

\author{
Claudio Pierantoni \\ UNIVERSIDAD DE CHILE
}

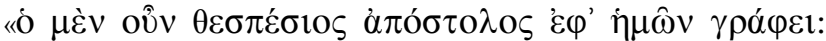

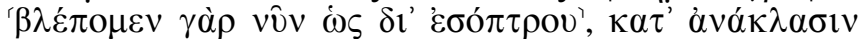

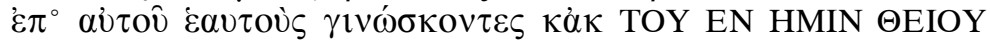

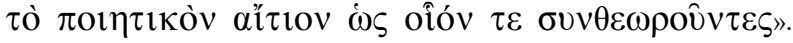

Clemente Alejandrino ${ }^{1}$
\end{abstract}

Nos proponemos analizar el concepto de verbum formabile («hoc formabile nondumque formatum»), que San Agustín discute en una importante página del De Trinitate $(\mathrm{XV}, \mathrm{xv}, 25)^{2}$.

Esta misteriosa realidad, presente a nuestra mente de modo inicialmente informe y, sin embargo, verdadero y real, es un verbo todavía silencioso, pero necesario presupuesto (a prior ) de aquella actividad interior, que es el pronunciamiento del verbum cordis: la palabra humana, ya en parte

1 «El divino Apóstol, refiriéndose a nosotros mismos, escribe: "Al presente, vemos como en un espejo", en cuanto que nos conocemos a nosotros mismos según ese reflejo, y porque contemplamos, cuanto es posible, la causa creadora a partir del

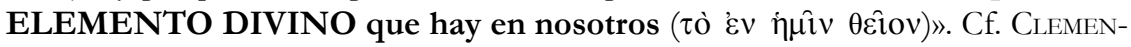
TE DE Alejandría, Stromata I.xix.94.4 (Madrid 1996) 273. Anota el P. Merino: «Con otras palabras: "nos conocemos a nosotros mismos por el rayo que se refleja sobre el espejo", que somos nosotros mismos.» (Clemente DE Alejandría, Stromata, nota 20). Los evidenciados son míos.

2 S. Aurelit Augustini, De Trinitate Libri XV, aux. Fr. Glorie, Corpus Christianorum LA (Turnholti 1968). Para la traducción española hemos utilizado libremente el volumen de las Obras completas de San Agustín, V: La Trinidad, traducción, introducción y notas de L. Arias (Madrid 1985). 
formada e inteligible, que nace de este verbo inicial informe, al contacto del hombre con el mundo real, mediante la actividad de la cogitatio.

Interpretaremos el pasaje a la luz del contexto de los capítulos anteriores del libro XV, donde precisamente el Doctor de Hipona analiza la generación del verbum cordis en el hombre y la pone en paralelo con la generación del Verbo divino. Buscaremos así comprender más a fondo la armonía entre la fundamental doctrina agustiniana de la iluminación de la mente con la doctrina, igualmente fundamental, de la imagen trinitaria en el hombre.

El concepto se profundizará, también, a la luz de la doctrina del acies animi, la mirada de la mente, en la dinámica entre la memoria y la formación de las species inteligibles, analizada en el libro XI del mismo De Trinitate.

Mediante el análisis y la comparación de estos textos, será posible percibir de modo más claro y evidente la íntima relación, y al mismo tiempo, la infinita diferencia, entre el misterioso «verbo del corazón» humano y el eterno Verbo Creador.

El libro XV del De Trinitate representa una de las cumbres -quizás la cumbre más alta- alcanzada por el genio especulativo del Hiponense. Entre estas páginas, aquellas dedicadas específicamente al verbum cordis humano como imagen del Verbo divino (XV,x,17 - xv,25) están sin duda entre las más densas y significativas.

Analizando esta sección, vemos que, en primer lugar, Agustín nota la distinción entre (1) la palabra pronunciada físicamente, en voz alta, y (2) la palabra solo pensada, es decir, de la cual se piensa el sonido, bajo la forma de escucha silenciosa (mental), que, sin embargo, se refiere todavía a una realidad externa (sensible) y convencional, como lo demuestra el hecho que es distinta en los distintos idiomas.

Pero hay que transcender esta realidad («transeunda sunt haee»): más allá que esta, (3) hay una palabra más silenciosa, que es aquella de la realidad inteligible, vista por el pensamiento en la visio cogitationis ${ }^{3}$ : esta realidad es la que propiamente merece ser llamada verbum, mientras que la palabra

3 Platón, en el Sofista, contrapone al «diálogo interior y sin voz del alma consigo misma

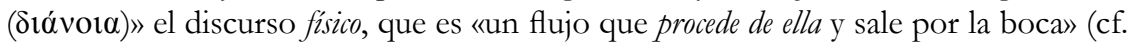
Platón, Sofista 263 e). 
sensible no es sino vox verbi. Para distinguirla con claridad de esta última, Agustín la llamará, por tanto, verbum cordis, o verbum mentis ${ }^{4}$.

El verbum cordis, producto de la cogitatio humana, es claramente un verbo bumano bien distinto del Verbo divino; sin embargo, se revela como semejante a Aquel, como imagen en un espejo (per speculum), aunque oscuro (in aenigmate $)^{5}$.

Ahora bien, vemos que entre la génesis del verbum cordis, imagen del Verbo divino, y la generación del mismo Verbo en la eternidad, puede verse una profunda relación, que nos lleva hasta el punto más misterioso y más neurálgico de la metafísica agustiniana. Y, podríamos agregar, de la metafísica en general.

En efecto, el verbum cordis, aunque inicialmente informe y silencioso, si se le ve en relación con el lenguaje formado (tanto sensible como mental), reenvía en realidad a un Silencio más profundo y original. Como verbo humano, él es siempre pronunciado en el tiempo, y la visio cogitationis (la visión del pensamiento humano), no es eterna, sino contingente: viene y va, y debe siempre recurrir a la fuente de la memoria, la fuente interior y superior, de la cual el mismo verbo se genera.

El patrimonio de la memoria, por cierto, es esencialmente verdadero, pues la memoria misma -en el sentido técnico agustiniano- aunque se experimente en un proceso histórico, no tiene su origen y raíz en el mundo temporal, sino que contiene lo que es transcrito directamente «de la verdad incorpóreaı ${ }^{6}$, es decir, de aquellas realidades inteligibles, a las cuales la mente humana está esencialmente unida por disposición creacional ${ }^{7}$.

La cogitatio, entonces, aun operando en el tiempo, está en condición de generar un verbo verdadero, del tesoro de la memoria ${ }^{8}$.

4 Véase a este propósito el análisis fundamental del Comentario de Agustín al Evangelio de San Juan (Tractatus in Iohannem I,8-9). El punto esencial se resume en la frase: «est vere verbum quod vere spiritaliter dicitur, illud quod intelligis de sono, non ipse sonus».

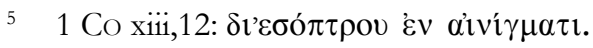

6 XII, xiv, 23: «ubi primum [scil. cogitatio] invenerat, in illa incorporea veritate, unde rursus quasi descriptum in memoria figeretur».

7 XII,xv,24: «rebus intellegibilibus, naturali ordine disponente Conditore subiuncta, sic ista videat in quadam luce sui generis incorporea».

8 XV,xii,22: «Haec igitur omnia, et quae per se ipsum, et quae per sensus sui corporis, et quae testimoniis aliorum percepta scit animus humanus, thesauro memoriae 
A tal generación concurren, por tanto, dos elementos:

a) la impresión o información sensible, a la cual se convierte la atención (cogitatio) humana;

b) la intelección de los datos sensibles, gracias a aquella lux sui generis incorporea, que propiamente constituye la base ontológica de las realidades inteligibles percibidas por el hombre, confiriéndoles aquel carácter de universalidad y necesidad, que innegablemente percibimos.

Detrás de tal lux sui generis incorporea, ciertamente Agustín ve la acción misteriosa del Verbo divino; sin embargo, es muy digno de observación que no se refiera aquí al Verbo como Persona. Nos parece que aquí, precisamente, nuestro doctor supera de manera clara e inequivocable el riesgo de ambigüedad que, en obras anteriores, podía darse en ciertas expresiones que identificaban directamente la luz inteligible con el Verbo?.

En la memoria como tal, por tanto, como patrimonio de verdad, está presente un silencio más profundo que aquel del verbum cordis pronunciado en la vida temporal: pero hemos visto que la memoria misma se constituye en el hombre por el contacto con las realidades inteligibles presentes en la luz, o, por decirlo con una famosa expresión agustiniana, con aquello que el hombre lee «en el libro de aquella lur, que se llama verdad» $»^{10}$.

Pero, si el contenido inteligible percibido por la mente humana, aunque oscuramente, es un reflejo de la Eterna Verdad, la operación de lectura, que, unida a la experiencia sensible, da lugar al patrimonio de la memoria

condita tenet, ex quibus gignitur verbum verum, quando quod scimus loquimur, sed verbum ante omnem sonum, ante omnem cogitationem soni. Tunc enim est verbum simillimum rei notae, de qua gignitur et imago eius, quoniam de visione scientiae visio cogitationis exoritur, quod est verbum linguae nullius; VERBUM VERUM DE RE VERA, nibil de suo habens, sed totum de illa scientia de qua nascitur».

9 Cf., por ejemplo, Soliloquia I,i,2: «Deus, qui nisi mundos verum scire noluisti» (véase la interesante retractación de esta frase en: Retractationes I,4); De vera religione xxxi,57: «Nec iam illud ambigendum est, incommutabilem naturam, quae supra rationem animam sit, Deum esse; et ibi esse primam vitam et primam essentiam, ubi est prima sapientia. Nam haec est illa incommutabilis veritas, quae lex omnium artium recte dicitur et ars omnipotentis artificis». Mediante las aclaraciones del De Trinitate, podríamos decir que la ley de las artes (humanas) es la luz del intelecto, la cual ciertamente depende de la ars divina creadora, pero no puede identificarse con ella sin más. Cf. también Confessiones VII,x,16 (ver infra, nota 20).

10 XIV,xv,21: «in libro lucis illius quae veritas dicitur». 
ya formada - de la cual se genera el verbum cordis- es aun una operación temporal y, en el sujeto temporal, es accidental. Agustín, por tanto, se dirige, en el pasaje que constituye el centro de nuestra atención, a considerar aquel silencio humano originario y esencial, que antecede no solo la cogitatio, que genera el verbo de la memoria, sino también la primitiva cogitatio, que fue necesaria a formar el patrimonio de la memoria misma.

Se trata específicamente, aquí, de la possibilitas cogitationis, de la posibilidad misma del pensar, en sí considerada. Leamos el pasaje:

«Las cosas, empero, que se conocen de tal suerte, que nunca es posible olvidar, por estar siempre presentes y pertenecer a la misma naturaleza del alma (ad ipsius animi naturam pertinent), como el saber que vivimos -mientras permanece el alma, permanece esta ciencia, y como el alma siempre permanece, permanece esta ciencia-; esto y otras cosas similares, si existen, en las que más bien se ha de intuir la imagen de Dios, aunque siempre se conocen (semper sciuntur), no siempre pensamos en ellas (non semper cogitantur), y así resulta difícil averiguar en qué sentido se llama eterno el verbo que nace de esta ciencia, ya que nuestro verbo se pronuncia mediante nuestro pensamiento (verbum nostrum nostra cogitatione dicatur).

Eterno es al alma el vivir, eterno el conocimiento de su vivir, pero no es eterno el pensar en su vida, o pensar en el conocimiento de su vivir, porque, cuando comienza a pensar en unas cosas, deja de pensar en otras, sin cesar de conocer. De donde se infiere que, si es posible una ciencia eterna en el alma, no es eterno el pensamiento de esa ciencia; y, si nuestro verbo íntimo y verdadero solo puede ser expresado por nuestro pensamiento, se sigue que únicamente Dios tiene un Verbo sempiterno y coeterno a Él. A no ser que se diga que la misma POSIBILIDAD DE PENSAR (possibilitas cogitationis) es ya verbo tan perpetuo, como perpetua es la ciencia, porque aquello que se conoce, aunque no se piense, PUEDE, sin embargo, SER PENSADO.

Pero, ¿cómo puede ser verbo lo que en la visión del pensamiento aún no se ha formado (nondum in cogitatione visionis formatum est)? ¿Cómo ha de ser semejante a la ciencia que le da el ser, si aún no posee su FORMA, y se denomina verbo tan solo porque PUEDE tenerla? Esto equivaldría a decir que se ha de llamar verbo, porque PUEDE ser verbo.

Mas, ¿qué es lo que PUEDE ser verbo, y por eso es ya digno del nombre de verbo? ¿Qué es, repito, ESTO FORMABLE, AÚN NO 
FORMADO, sino un algo de nuestra mente, que nosotros con antojo voluble lanzamos de aquí para allá, cuando pensamos ahora en una cosa, y después en otra, según la descubrimos o nos sale al encuentro? Y llega a ser VERBO VERDADERO, cuando aquello que, como dije, lanzamos ${ }^{11}$ con voluble movimiento, llega a lo que conocemos, y de allí toma su forma (inde formatur), recibiendo su completa semejanza (omnimodam similitudinem); de modo que, tal como la cosa se conoce, así también se piense y SE PRONUNCIE EN EL CORAZÓN (in corde dicatur), es decir, sin palabra, ni pensamiento de palabra, cosas que, por cierto, son particulares de un idioma.

Por consiguiente, aun concediendo -para no dar la sensación de que disputamos sobre palabras- que se puede llamar VERBO a aquel algo de nuestra mente que puede formarse de nuestra ciencia antes de su formación, porque es ya, por decirlo así FORMABLE, ¿quién no ve cuan inmensa es la diferencia con el Verbo de Dios, que es EN LA FORMA DE DIOS ${ }^{12}$, de tal modo que no es primero formable y después formado, ni en algún momento puede ser informe, sino que es forma simple y simplemente igual a Aquél de quien subsiste, y al cual es admirablemente coeterno?

(XVI.) Por lo cual, para que no se crea que en Dios existe algo voluble, como si, siendo Verbo, pudiera recibir y volver a tomar una forma que pronto pudiera perder, y sufrir evolución en su amorfía, aquel Verbo divino no se llama 'pensamiento de Dios' (cogitatio Dei), [...] sino 'Verbo de Dios' (Verbum Dei). [...]

Ni aun cuando seamos semejantes a Él, [...] no se ha de comparar con aquella simplicidad maravillosa, donde no existe nada formable, ni formado, ni reformado, sino FORMA; ni informe, ni formada, sino SUSTANCIA (substantia) eterna e inmutable» ${ }^{13}$.

11 «Illud quod nos dixi volubili motione iactare». La traducción de la BAC traduce erróneamente: «aquello que dije nos lanzaba». El sujeto de iactare es claramente nos: «nosotros lanzamos».

12 «In forma Dei exsistens» (Fil 2,5).

$13 \mathrm{XV}, \mathrm{xv}, 25$ : «Illa etiam quae ita sciuntur, ut numquam excidere possint, quoniam praesentia sunt, et ad ipsius animi naturam pertinent, ut est illud quod nos vivere scimus (manet enim hoc quamdiu animus manet, et quia semper manet animus, et hoc semper manet): id ergo et si qua reperiuntur similia, in quibus imago Dei potius intuenda est, etiamsi semper sciuntur, tamen quia non semper etiam cogitantur, quomodo de his dicatur verbum sempiternum, cum verbum nostrum 
Agustín está, aquí, en búsqueda de un verbo que sea verdaderamente nuestro, humano, pero al mismo tiempo, de algún modo, refleje la eternidad que es propia de la scientia, de aquella realidad inteligible, inmutable, que por esencia está presente a la mente, pero no parece poder decirse propiamente nuestra, si no pasara por la cogitatio («verbum nostrum, nostra cogitatione dicatur»). Por eso, con el fin de encontrar algo que sea nuestro, se dirige a aquella posibilidad del pensar (possibilitas cogitationis), que finalmente bautiza verbum formabile. Esto es «algo de nuestra mente», aquello que «con movimiento voluble podemos dirigir» a diferentes objetos, $\mathrm{y}$ que llega, mediante la información recibida del mundo externo por los sentidos, a ser FORMADO, y a recibir la completa semejanza (omnimodam

nostra cogitatione dicatur, invenire difficile est. Sempiternum est enim animo vivere, sempiternum est scire quod vivit: nec tamen sempiternum est cogitare vitam suam, vel cogitare scientiam vitae suae; quoniam cum aliud atque aliud coeperit, hoc desinet cogitare, quamvis non desinat scire. Ex quo fit, ut si potest esse in animo aliqua scientia sempiterna, et sempiterna esse non potest eiusdem scientiae cogitatio, et VERBUM VERUM NOSTRUM INTIMUM nisi nostra cogitatione non dicitur, solus Deus intellegatur habere Verbum sempiternum sibique coaeternum. Nisi forte dicendum est, IPSAM POSSIBILITATEM COGITATIONIS, quoniam id quod scitur, etiam quando non cogitatur, potest tamen veraciter cogitari, verbum esse tam perpetuum, quam scientia ipsa perpetua est. Sed quomodo est verbum, quod nondum in cogitationis visione formatum est? Quomodo erit simile scientiae de qua nascitur, si eius non habet formam, et ideo iam vocatur verbum, quia potest habere? Tale est enim ac si dicatur, ideo iam vocandum esse verbum, quia POTEST ESSE VERBUM. Sed quid est quod potest esse verbum, et ideo iam dignum est verbi nomine? Quid est, inquam, HOC FORMABILE NONDUMQUE FORMATUM, nisi quiddam mentis nostrae, quod hac atque hac volubili quadam motione iactamus, cum a nobis nunc hoc, nunc illud, sicut inventum fuerit vel occurrerit, cogitatur? Et tunc fit verum verbum, quando illud quod nos dixi volubili motione iactare, ad id quod scimus pervenit, atque inde formatur, eius OMNIMODAM SIMILITUDINEM capiens; ut quomodo res quaeque scitur, sic etiam cogitetur, id est, sine voce, sine vocis cogitatione, quae profecto alicuius linguae est, sic in corde dicatur. Ac per hoc etiam si concedamus, ne de controversia vocabuli laborare videamur, iam vocandum esse verbum quiddam illud mentis nostrae quod de nostra scientia formari potest, etiam priusquam formatum sit, quia iam, ut ita dicam, formabile est; quis non videat, quanta hic sit dissimilitudo ab illo Dei Verbo, quod in forma Dei sic est, ut non antea fuerit formabile postque formatum, nec aliquando esse possit informe, sed sit forma simplex et simpliciter aequalis ei de quo est, et cui mirabiliter coaeterna est. (xvi.) Quapropter ita dicitur illud Dei Verbum, ut Dei cogitatio non dicatur, ne aliquid esse quasi volubile credatur in Deo, quod nunc accipiat, nunc recipiat formam, ut verbum sit, eamque possit amittere, atque informiter quodam modo volutari». 
similitudinem) de las cosas conocidas. Por eso, el hombre puede engendrar en sí mismo un verbo verdadero.

Notemos que aquí, aunque con terminología diferente, Agustín ya expresa con perfecta claridad el contenido de lo que la escolástica medieval llamará adaequatio intellectus et rei. Donde las cosas mismas son consideradas causas de la verdad ${ }^{14}$ que está en el entendimiento humano, mientras que el entendimiento divino es causa de las cosas creadas.

Ahora bien, este «algo de nuestra mente», diferente de la scientia, que es la realidad inteligible considerada en sí misma, no puede ser sino aquel misterioso espejo - del que hablaba ya San Pablo- que recibe en sí la imagen refleja de aquella realidad inteligible, que está presente a la mente y que puede siempre ser consultada mediante el acto de la cogitatio, pero que ya desde el primer momento la ilumina, aunque sea de un modo todavía indeterminado (《informe»).

La imagen bíblica del espejo tiene en Agustín, en la huella de la tradición platónica, un equivalente terminológico técnico: se trata, en sustancia, de la «vista del alma» (acies animi), de la que ya él ha hablado en el libro XI:

\section{«acies autem animi quae reminiscendo FORMATUR $»^{15}$.}

La «vista del alma», siguiendo la metáfora platónica, no puede ver los seres por su mera presencia física, que actúa sobre los sentidos; las sensaciones, en efecto, y aun los efectos mentales -como efectos físicos en el cerebro- no serían de ningún modo suficientes a dar razón de la universalidad y necesidad («eternidad») del conocimiento, que es tal, aun cuando este se aplique a los entes contingentes. Por tanto, es necesario postular, como causa de la verdad, como la experimentamos en nuestro entendimiento, la

14 Más precisamente, habría que decir concausas, pues causa principal de la verdad en el intelecto humano es, para los Escolásticos, tal como para Agustín, la luz del intelecto. Véase por ejemplo, en S. Tomás De AQuino, la conclusión de la responsio de la Quaestio XI De Veritate (De magistro): «Huiusmodi autem rationis lumen, quo principia huiusmodi nobis sunt nota, est nobis a Deo inditum, quasi quaedam SIMILITUDO INCREATAE VERITATIS in nobis resultans. Unde, cum omnis doctrina humana efficaciam habere non possit nisi ex virtute illius luminis, constat quod SOLUS DEUS EST QUI INTERIUS ET PRINCIPALITER DOCET, sicut natura interius et principaliter sanat». (Cf. también infra, nota 19).

15 XI, vii, 11. 
presencia de una luz especial ${ }^{16}$, aquella lux sui generis incorporea, que ya hemos encontrado en el libro $\mathrm{XII}^{17}$.

Solamente una luz especial, en el sentido de que no se trata de una realidad que proviene del mundo contingente, $y$, al mismo tiempo, se diferencia de la presencia real del Verbo divino (siendo de este solo un reflejo indirecto), puede dar razón del carácter ontológico peculiar de los contenidos inteligibles: humanos («verba nostra»), y, sin embargo universales, por tanto, reflejo de la realidad eterna.

Esta acies, que podemos, por tanto, identificar con el verbum formabile, es precisamente aquello que nosotros «lanzamos ahora aquí, y después allá», hacia diferentes objetos, que nos devienen inteligibles por la luz especial interior, permitiéndonos apropiarnos de ellos; pero tal acies existe ya en sí misma, anteriormente a la cogitatio: ya, por así decirlo, informada de un modo indeterminado.

Por tanto, hay en nosotros, en nuestra misma esencia, algo así como un espejo silencioso, al que podemos llamar potentia cogitationis: decimos potentia, pues la possibilitas, de la que habla Agustín en este caso, es propia esencialmente de un sujeto real: por tanto, no se trata de una mera posibilidad abstracta, sino de una verdadera y real potencia. Se trata, en suma, de un espejo abierto a la verdad esencialmente, es decir, anterior e independientemente de sus operaciones contingentes (accidentales).

Ahora bien, hay que reflexionar que la forma que se refleja en este espejo, la «forma de la verdad» (también llamada facies veritatis, el «rostro

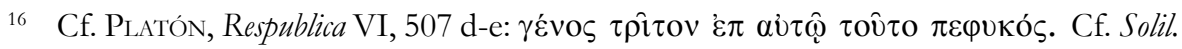
I,viii, 15 .

17 Cf. XII,xv,24 (cf. supra, nota 5). La interpretación de esta expresión («sui generis») ha sido objeto de una secular discusión, que no podemos aquí resumir. Baste señalar que, traduciendo con «luz especial», nos adherimos sustancialmente a la posición de S. Buenaventura, que amplía la interpretación de S. Tomás, quien proponía identificar sin más el género de la luz con el género del alma, y consideraba que lux sui generis significaría «del mismo género del alma». Buenaventura, sin rechazar esta interpretación - pues hay, en cierto sentido, un parentesco entre ambos géneros, por cuanto espirituales («no materiales»)- considera, sin embargo, importante subrayar que tal luz es, más bien, «de un género propio» (precisamente, evidenciando que tal luz es superior al alma). Una excelente discusión del problema puede verse en M. F. SCIACCA, Sant'Agostino (Palermo 1941), «Note finali», 367-368. Sciacca también se adhiere sustancialmente a la interpretación de S. BuEnaventura. 
de la verdad», en el vocabulario agustiniano ${ }^{18}$ ), es forma en sentido objetivo, lo cual es importantísimo para entender que la realidad inteligible, que el sujeto refleja, es superior a la esencia real del sujeto mismo.

Expliquemos mejor: la realidad inteligible, o, lo que es lo mismo, la luz del intelecto (que incluye, en potencia, todas las especies abstractas y en general, todos los inteligibles ${ }^{19}$ ) perfecciona al intelecto mismo, por cuanto el sujeto humano va conociendo las cosas creadas que impresionan sus sentidos, así como aquellas de las que tiene noticia indirecta: el sujeto entra en contacto con lo inteligible, y puede decirse que recibe forma de él. Pero, no en el sentido -este es el punto más importante- de que él (su ser real) se confunda con la esencia real de las cosas externas como tales, lo cual implicaría el absurdo de que el sujeto humano debería, conociendo la esencia de un ente inferior a él mismo, abajarse al nivel de este; sino, por el contrario el hombre, contemplando la verdad de las cosas, se eleva a una realidad inmutable que se halla por sobre su mente ${ }^{20}$, pues ella -justamente en su inmutabilidad- revela su ser necesariamente un reflejo de aquella Eterna Sabiduría que crea las cosas temporales, sin por eso confundirse con el-

18 Cf. Soliloquia II,xx,35.

19 Pues todas están de algún modo grabadas «en el libro de aquella LUZ, que se llama VERDAD», que de la eterna Sabiduría se imprime en el alma, tal como el sello que de un anillo marca la cera, sin por eso dejar el anillo: «non migrando, sed tamquam IMPRIMENDO transfertur; sicut IMAGO ex anulo et in ceram transit et anulum non relinquit». (cf. XIV,xv,21). Lo mismo, con síntesis magistral, expresa S. TomÁs DE AQUiNo: «Dicendum quod in luce Primae Veritatis omnia intelligimus et iudicamus, inquantum ipsum LUMEN INTELLECTUS nostri, sive naturale sive gratuitum, nihil aliud est quam quaedam IMPRESSIO VERITATIS PRIMAE». (S. Theol. I' ${ }^{\mathrm{a}}$ q. 88 a. 3 ad 1). Cf. supra, nota 12.

20 Concepto clarísimo en Agustín ya desde los diálogos filosóficos, pero magistralmente expresado sobre todo en las Confesiones, que resumen también la experiencia mística que en Agustín acompañó y guió tan grande descubrimiento intelectual: «admonitus redire ad memetipsum, intravi in intima mea duce te et potui, quoniam factus es adiutor mens. Intravi et vidi qualicumque oculo animae meae supra eumdem oculum animae meae, supra mentem meam lucem incommutabilem, non hanc vulgarem et conspicuam omni carni nec quasi ex eodem genere grandior erat, tamquam si ista multo multoque clarius claresceret totumque occuparet magnitudine. Non hoc illa erat, sed aliud, aliud valde ab istis omnibus. Nec ita erat supra mentem meam, sicut oleum super aquam nec sicut caelum super terram, sed superior, quia ipsa fecit me, et ego inferior, quia factus ab ea. Qui novit veritatem, novit eam, et qui novit eam, novit aeternitatem. Caritas novit eam. $\mathrm{O}$ aeterna veritas et vera caritas et cara aeternitas! Tu es Deus meus, tibi suspiro die ac nocte». (VII,x,16). 
$\operatorname{las}^{21}$. Y es así, propiamente, que el sujeto puede recibir, ya desde su inicio intelectualmente informe y gradualmente, hasta su perfecto crecimiento y madurez en Cristo, aquella forma que es propia del Verbo Creador.

En base a lo anterior, podemos ahora ver más claramente la profundización que Agustín realiza en el ámbito de la doctrina cristiana tradicional del hombre como imagen de Dios, en perfecta armonía con la doctrina de la iluminación del intelecto.

Hay en el hombre, como decíamos, un primordial espacio abierto, pero no una pura nada, ni una mera potencialidad abstracta, sino una potencia real de pensar: o, más bien dicho, el hombre mismo es esta potencia de pensar: él es, por tanto, ya en sí mismo, de algún modo, verbo, aunque sea de un modo todavía incipiente, por tanto, formable: es ya una verdadera actualidad inteligente, que por naturaleza está unida a la actualidad inteligible de la lux incorpórea. Como tal, recibirá forma y crecimiento, por cuanto el sujeto mismo es capaz, con el concurso de su libre voluntad, de generar en sí mismo una imagen del Verbo en progresivo perfeccionamiento.

A confirmación de lo anterior, recordemos que ya en el libro XI, hablando de la «vista del alma» (acies animi), que se forma «de la memoria, recordando» (ex memoria, reminiscendo), Agustín afirma que ella preexiste necesariamente a la formación de la memoria misma ${ }^{22}$ : por tanto, la species impressa, que se expresa de la memoria y da forma a la acies cogitantis, non es, propiamente hablando, vera proles, ni aquella (la memoria), vera parens, sino que, esta, quasi parens, aquella, quasi proles ${ }^{23}$.

Esto confirma, por un lado, que no hay, en el sujeto humano anteriormente a sus operaciones racionales, una mera y pasiva tabula rasa, sino, como decíamos, una actualidad esencialmente inteligente. Pero es claro también, por otro lado, que no se trata de una generación en sentido originario y absoluto, sino de una participación en una generación de or-

21 Además que en la Creación, también se expresa el mismo concepto a propósito de la Encarnación, mediante el adverbio ảov $\gamma \chi u ́ \tau \omega \varsigma$ «sin confusión» (CONCILIO DE CALCEDonia, 451, cf. Denzinger-Hünermann, 302).

22 XI,vii,11: «Acies autem animi quae reminiscendo formatur, erat etiam priusquam corpus quod meminimus videremus; quanto magis priusquam id memoriae mandaremus?».

$23 \mathrm{XI}, \mathrm{vii}, 11$ : «neque illa vera parens, neque ista vera proles est [...]. Sed et illa quasi parens, et ista, quasi proles». Cf. Tractatus in Iohannem I,9: «Si tu potes habere verbum in corde tuo, tamquam consilium natum in mente tua, ut mens tua pariat consilium, et insit consilium quasi proles mentis tuae, quasi filius cordis tui». 
den superior a su esencia creada: es claro, justamente, por cuanto el verbo bumano generado, no es una emanación del sujeto bumano, de su esencia propia (como ocurre en la Trinidad divina ${ }^{24}$ ), sino algo que lo lleva más allá de sí mismo, lo perfecciona: en efecto, para generar este verbo, es necesario, por un lado, el concurso continuo de aquella actualidad inteligible que es la luz inmutable, que resplandece a la interioridad del hombre (es decir, el concurso divino, aunque "natural", distinto de aquella conjunción personal y real que llamamos "Gracia”); y, por otro lado, el concurso de las cosas creadas, con su acción sensible. Aquí, justamente, está la maravilla de esta generación: es decir, que ella produce, o, mejor dicho, coopera a producir, con un acto humano voluntario y natural (la cogitatio), el perfeccionamiento del hombre mismo.

Ahora bien, que la essentia veritatis, contemplada por la mente, sea superior al hombre y lo perfeccione, es doctrina antigua y constante en Agustín; por otra parte, también es doctrina ya adquirida en Agustín la transformación del hombre interior por influjo de la Gracia.

Pero la profundización característica y específica de esta página consiste en la observación que, prescindiendo ya sea (1) de las operaciones accidentales de la inteligencia, ya sea (2), del consentimiento a la acción divina de la Gracia, existe algo de nuestra mente, que recibe propiamente su forma objetiva de la Forma, simple y perfecta, del Verbo divino.

Existe, por tanto, en la trinidad humana natural, formada por memoria, intellegentia, voluntas, un elemento divino, que objetivamente la informa: no solamente el verbum cordis pronunciado y formado con la actividad voluntaria, sino que ya, en su principio silencioso, el verbum cordis formabile: este es, por consiguiente, la impresión más esencial y originaria, en el ser humano, del verbo eternamente engendrado por el Padre.

Esta es, entonces, la imagen divina originaria en el hombre, que no puede adquirirse ni perderse, ni depende de la edad, de la salud física o mental, ni de cualquier otra cosa contingente.

Profundizando en la doctrina del conocimiento, Agustín nos ofrece aquí una profundización esencial en la doctrina del hombre como trinidad hecha a imagen de Dios.

24 Recordemos la expresión antes vista: «verbum verum de re vera», en claro paralelismo, y contraste al mismo tiempo, con la fórmula del Símbolo niceno: «deum verum de deo vero». 
Es claro, a la luz de estas páginas del De Trinitate, que el hombre, respecto de Dios, no puede ser visto simplemente como imagen de un modelo, que se coloque frente a él de un modo estático o meramente pasivo, sino que es un ser llamado a participar del dinamismo generativo interno de la Trinidad divina, como espejo que por naturaleza refleja y contempla, aunque de modo indirecto, e inicialmente oscuro e indeterminado, la generación de la eterna Verdad.

$\mathrm{Y}$ este dinamismo intelectual y contemplativo, lo repetimos una vez más para concluir, es propio del ser humano independientemente de sus operaciones históricas, en el tiempo de la vida terrenal: es algo de su esencia, que funda su infinita dignidad, independientemente del momento y del grado de su desarrollo natural, cultural o espiritual, y de cualquier accidente de la vida. 
Resumen: En este trabajo se analiza el concepto de «verbum formabile», que San Agustín discute en De Trinitate XV,xv,25. Esta realidad, presente a nuestra mente de modo inicialmente informe y, sin embargo, verdadero y real, es un verbo todavía silencioso, pero necesario presupuesto de aquella actividad interior, que es el pronunciamiento del verbum cordis, que es la palabra humana, ya en parte formada e inteligible, que nace de este verbo inicial informe, al contacto del hombre con el mundo real, mediante la actividad de la cogitatio. La generación del verbo en la mente humana, en efecto, exige, en lo interior del ser humano, no solo una mera y pasiva tabula rasa, sino una actualidad esencialmente inteligible, participación de la Verdad eternamente generada, aunque en nuestra visión, inicialmente indeterminada. El verbo de la mente humana no es, por tanto, el mero producto de la esencia finita del hombre, sino participación dinámica en la acción infinita que trae su origen de la actividad creadora de la Trinidad divina, y cuyo reflejo en el cosmos sensible se proyecta realmente, aunque de modo enigmático, en el maravilloso espejo interior de la mente.

Palabras clave: Agustín, De Trinitate, verbum formabile, gnoseología.

Abstract: This work analyzes the concept of "verbum formabile", that Saint Agustine discusses in De Trinitate $\mathrm{XV}, \mathrm{xv}, 25$. Such reality is present to our mind in an initially "formless", but nevertheless, true and real way: it is a "silent" word, but a necessary presupposition of that inner activity that is the pronouncement of the "verbum cordis", which is the human word, partly already formed and intelligible, that is born from that initial formless word, with man's contact with the real world, by the activity of the "cogitatio". In fact, the word's generation in the human mind needs, in man's interior activity, not a mere passive "tabula rasa", but an intelligible actuality, a participation in the eternally generated Truth, although initially undetermined to our vision. The word of the human mind is not, therefore, the mere product of the finite human essence, but a dynamic participation in that infinite action that takes its origin in the creative activity of the divine Trinity, whose reflection in the empirical universe projects itself really, though enigmatically, in the marvelous interior mirror of the mind.

Key words: Augustine, De Trinitate, verbum formabile, gnosiology. 